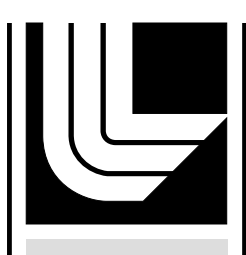

LAW RENCE LIVERMORE N A T IO N A L LABORATORY

\title{
Motion and Rotation of Small Glissile Dislocation Loops in Stress Fields
}

W. G. Wolfer, T. Okita

October 7, 2003

Physical Review Letters 
This document was prepared as an account of work sponsored by an agency of the United States Government. Neither the United States Government nor the University of California nor any of their employees, makes any warranty, express or implied, or assumes any legal liability or responsibility for the accuracy, completeness, or usefulness of any information, apparatus, product, or process disclosed, or represents that its use would not infringe privately owned rights. Reference herein to any specific commercial product, process, or service by trade name, trademark, manufacturer, or otherwise, does not necessarily constitute or imply its endorsement, recommendation, or favoring by the United States Government or the University of California. The views and opinions of authors expressed herein do not necessarily state or reflect those of the United States Government or the University of California, and shall not be used for advertising or product endorsement purposes. 


\title{
Motion and Rotation of Small Glissile Dislocation Loops in Stress Fields
}

\author{
W.G. Wolfer and T. Okita \\ Chemistry and Materials Science, Lawrence Livermore National Laboratory, \\ Livermore, CA 94550-9234
}

We derive here for the first time the combined motion and rotation of small prismatic dislocation loops in stress fields. When the applied torque is balanced by the self-torque of the loop, we show how the solution can be obtained for the loop orientation, and how this orientation affects the glide force on the loop.

Atomistic computer simulations of small clusters of self-interstitials have revealed that these clusters are highly mobile along certain crystallographic directions. Their thermal mobility and Brownian motion along these directions rapidly decreases, however, as the size of the cluster increases. A review of these computer simulations has been provided by Osetsky et al. [1], and more recent studies are given by Marian et al. [2]. All these studies have shown that the activation energy for cluster diffusion reaches a saturation value, while the pre-exponential factor continues to decline with the cluster or loop size. The diffusion of loops containing more than one hundred interstitials becomes too slow to be quantified with molecular dynamics simulations. Nevertheless, since the activation energy for migration becomes nearly independent of the loop size, they remain very mobile if forces act on them, even though their Brownian motion becomes insignificant. Such forces naturally exist in real crystal due to internal stress fields originating from other defects, in particular from dislocations.

Small clusters of self-interstitials, when no longer subject to rapid Brownian migration, are of course synonymous with small prismatic dislocation loops. When their Burgers vectors are aligned along one of the possible glide directions, they can move on onedimensional trajectories in response to the force exerted by their elastic interaction with stress fields of other defects. As we shall see, this force depends among other factors on the orientation of the dislocation loop, and in turn, the orientation is affected by the stress field. In other words, the motion of a small prismatic dislocation loop on its glide prism has three degrees of freedom, namely its position along the glide prism, and the orientation of its normal vector (normal on the loop plane) in relation to its Burgers vector or glide direction. The specification of the latter requires then two angles, hence a total of three degrees of freedom for one-dimensional motion.

Kroupa [3] has long ago pointed out that stress fields exert both a net force and a net torque on small dislocation loops that he derived from the Peach-Koehler force. Numerical studies of both force and torque have been carried out by Ghoniem et al.[4] for small prismatic dislocation loops interacting with a near-by dislocation, assuming however, a fixed orientation of the loop normal vector.

In order to go beyond these earlier studies, it is necessary to remove the constraint of a fixed orientation, and to derive a set of equations that satisfy both the balance of forces along the glide prism and the balance of force moments. The derivation of this complete set of the equations of motion and a method for their solution are given for the first time in this Letter.

We shall limit our considerations to over-damped motions of dislocation loops in which the kinetic energy is negligible compared to the potential energy. Gillis and Kratochvil 
[5] have justified this limitation when they showed that dislocations respond to changes in applied stress within 10 to 20 picoseconds. This means that for nearly all practical purposes, their velocity is determined by the balance of applied force, Peierls force, and the drag caused by phonon and electron scattering. Furthermore, the rotation of the normal vector of the loop to a sudden application of a torque will certainly occur with a similar, if not shorter response time. However, rotation is limited not so much by drag forces but by the increase in the strain energy of the loop itself.

With these considerations in mind, it is then adequate to write for the energy of a small prismatic dislocation loop of the interstitial type

$$
E=W \square \prod A A t(\mathbf{r}) \cdot \hat{\mathbf{n}} .
$$

Here, $W$ is the strain- or self-energy of the loop, and the second term is the interaction energy of the loop with the stress field originating from sources or defects other than the loop itself. For vacancy-type prismatic loops, the sign changes for the second term. The normal component of the traction vector field $t_{i}(\mathbf{r})=\square_{i j}(\mathbf{r}) b_{j}$ is integrated over the area of the loop whose orientation is defined by the unit normal vector $\hat{\mathbf{n}}$ and whose Burgers vector is $\mathbf{b}$. If the character of the dislocation loops remains the same, $\mathbf{b}$ is a constant vector. Perfect loops (but not faulted loops) may rotate, however, and $\hat{\mathbf{n}}$ remains to be determined. For this purpose, we need to specify both the self-energy of the loop as well as its shape and orientation. In order to give a more transparent derivation, the following simplifications are employed.

First, we assume that the traction vector field varies little over the area of the loop and we may replace it by an average value, say, its value at the loop center. Second, we assume that the shape of the loop is elliptical with a minor radius of $R_{0}$ and a major radius of $R$. Third, we consider an elastically isotropic solid and write for the self-energy of an elliptically shaped loop

$$
W=\frac{\square b^{2}}{4 \square(1 \square \square)} 4 R \mathbf{E}(k)(1 \square \square k) \ln \left(R_{0} / r_{0}\right) .
$$

Here, $\square$ is the shear modulus, $\square$ Poisson's ratio, $r_{0}$ is the dislocation core radius, and $\mathbf{E}(k)$ is the complete elliptic integral of the second kind. The form of the above equation is an adaptation of several analytical expressions or numerical results [6-9] which were obtained earlier by other researchers for the purpose of studying the loop energy as a function of its shape and rotation, and to determine the energy minimum, i.e. the orientation of the normal vector relative to the Burgers vector in the absence of stress. We note, that different researchers had arrived at different conclusions regarding this orientation, and we can replicate their various results by appropriate choice of the empirical parameter $\square$. For example, $\square=1 / 4$ results in an energy minimum for a loop when normal and Burgers vectors are parallel. This is the case considered most likely, and it will be adopted for the example given at the end. However, the general derivations given below is not restricted to this case. 
In equation (2), the loop orientation defines the parameter $k$ as

$$
k=1 \square(\hat{\mathbf{n}} \cdot \hat{\mathbf{b}})^{2}=1 \square \cos ^{2} \square
$$

where $\hat{\mathbf{b}}$ is the unit vector in the direction of the Burgers vector.

For convenience, we assumed also in equation (2) that when the loop shape is circular, $R=R_{0}, k=0$, and normal and Burgers vectors are parallel. We denote the area and energy of the circular loop by $A_{0}$ and $W_{0}$, respectively.

Rotation of the loop is accomplished when one side glides up and the opposite side down the glide cylinder. As a result, the shape becomes elliptical, increasing the loop area to

$$
A=\square R R_{0}=A_{0} / \cos \square
$$

and the loop circumference to $4 R \mathbf{E}(k)$. An elliptically shaped loop may in principle assume a circular shape again while maintaining its orientation. However, this requires pipe diffusion along its core, and this process can be neglected during glide motion.

The energy of a small dislocation loop in a stress field from a remote source must therefore be viewed as a doubly vector-valued scalar field given by

$$
E(\mathbf{r}, \hat{\mathbf{n}})=W(\hat{\mathbf{n}}) \square A(\hat{\mathbf{n}})(\mathbf{t}(\mathbf{r}) \cdot \hat{\mathbf{n}}),
$$

where $\mathbf{r}$ is the position of the loop center. The loop normal vector $\hat{\mathbf{n}}$ is in principle independent of $\mathbf{r}$, but it will become correlated by virtue of its dependence on the stress field, i.e. on $\mathbf{t}(\mathbf{r})$. To determine this correlation is the main objective of this paper.

The glide force on the loop is now given by

$$
F(\mathbf{r}, \hat{\mathbf{n}})=\square \square_{\mathbf{r}} E \cdot \hat{\mathbf{b}}=b A(\hat{\mathbf{n}}) \hat{n}_{i} \square_{i j, k}(\mathbf{r}) \hat{b}_{j} \hat{b}_{k}
$$

where the index $k$ after the comma indicates the partial derivative $\partial / \partial x_{k}$, and repeated indices imply a summation.

The net force moment, on the other hand, is obtained by evaluating $\hat{\mathbf{n}} \square \square_{\hat{\mathbf{n}}} E$. The rotational gradient of the loop energy is

$$
\square \square{ }_{\hat{\mathbf{n}}} W=2 \hat{\mathbf{b}} \sqrt{1 \square k} \frac{d W}{d k}=A(k) \square(k) \hat{\mathbf{b}}
$$

where

$$
\square(k)=\frac{W_{0}}{A_{0}} \frac{2}{\square k \sqrt{1 \square k}}\left\{\left(1 \square 3 k \square+2 k^{2} \square\right) \mathbf{E}(k) \square(1 \square k)(1 \square k \square) \mathbf{K}(k)\right\}
$$

and $\mathbf{K}(k)$ is the complete elliptic integral of the first kind. 
Equations (7) and (8) determine the intrinsic force moment of the dislocation loop itself, which we shall henceforth call the self-torque of a dislocation loop and denote as

$$
\mathbf{Q}(k)=\square \hat{\mathbf{n}} \square \square{ }_{\hat{\mathbf{n}}} W=\square(k)[\hat{\mathbf{n}} \square \hat{\mathbf{b}}]
$$

It is this force moment that restores the loop orientation to its minimum energy configuration in the absence of an applied force moment or torque. The external or applied torque is given by

$$
\mathbf{M}(k)=A(k)[\hat{\mathbf{n}} \square \mathbf{t}] \square A(k) \frac{(\mathbf{t} \cdot \hat{\mathbf{n}})}{(\hat{\mathbf{b}} \cdot \hat{\mathbf{n}})}[\hat{\mathbf{n}} \square \hat{\mathbf{b}}]
$$

Our result differs from the one originally obtained by Kroupa [3], as he assumed a loop area that remains constant with rotation. As a result, our first term in equation (10) contains an orientation-dependent loop area. In addition, we also obtain a new second term for the change in loop area as the loop rotates.

Neglecting frictional forces or activation barriers in loop rotation, the sum of self-torque and applied torque has to vanish, i. e.

$$
\hat{\mathbf{n}} \square \square_{\hat{\mathbf{n}}} E=\mathbf{Q}(k)+\mathbf{M}(k)=0
$$

Using eqs.(9) and (10), this equation can also be written as

$$
\left.[\hat{\mathbf{n}} \square \mathbf{t}]=[\hat{\mathbf{n}} \square \hat{\mathbf{b}}] \square(k)+\frac{(\mathbf{t} \cdot \hat{\mathbf{n}})}{(\hat{\mathbf{b}} \cdot \hat{\mathbf{n}})}\right]
$$

It follows from this equation that the loop normal vector $\hat{\mathbf{n}}$ lies in the same plane as $\hat{\mathbf{b}}$ and $\mathbf{t}$. This can be shown by forming the vector product of equation (12) with $\hat{\mathbf{b}}$; one obtains

$$
\left.\hat{\mathbf{n}} \square(k)+\frac{(\mathbf{t} \cdot \hat{\mathbf{n}})}{(\hat{\mathbf{b}} \cdot \hat{\mathbf{n}})} \square \hat{\mathbf{b}} \cdot \mathbf{t}\right]=[\square(k)(\hat{\mathbf{b}} \cdot \hat{\mathbf{n}})+(\mathbf{t} \cdot \hat{\mathbf{n}})] \hat{\mathbf{b}} \square(\hat{\mathbf{b}} \cdot \hat{\mathbf{n}}) \mathbf{t}
$$

The loop normal vector is indeed found to be the difference of two vectors, one proportional to the Burgers vector of the loop, the other proportional to the traction vector. Furthermore, it also follows from these vector equations that the normal vector can not lie between the two vectors $\mathbf{t}$ and $\hat{\mathbf{b}}$. Neither of these equations is suitable to determine quantitatively the orientation of the normal vector in the plane spanned by the Burgers and the traction vectors. For this purpose, it is better to use the absolute value of equation (12) together with the angular relationships easily verified from the diagram shown as insert in Figure 1: 


$$
\begin{aligned}
& \cos \square=\hat{\mathbf{n}} \cdot \hat{\mathbf{b}}=\sqrt{1 \square k} \\
& |\mathbf{t}| \cos \square=\hat{\mathbf{b}} \cdot \mathbf{t} \\
& |\mathbf{t}| \cos (\square \square \square=\hat{\mathbf{n}} \cdot \mathbf{t}
\end{aligned}
$$

Note, that the angle $\square$ is known, while $\square$ needs to be determined. Elementary manipulations lead to the final equation

$$
\operatorname{sign}(\square \sqrt{k(1 \square k)} \square(k)=|\mathbf{t}| \sin \square
$$

which must be solved numerically for $k$, and hence for the angle $\square$ between the Burgers and the normal vector. Note, that the normal vector $\hat{\mathbf{n}}$ may either lie on one or the other side of the vector $\hat{\mathbf{b}}$, corresponding to the two solutions $\pm \sqcup$ admitted by the first of the equations (14). One solution gives a stable orientation, the other one an opposed orientation in which the loop is in an unstable state.

To assist the reader interested in the use of the above equations, we shall apply them to the example displayed in Figure 1. A prismatic dislocation loop moves along its glide cylinder that is parallel to the glide plane and perpendicular to the line direction of a pure edge dislocation. Alternatively, one may consider the center of the prismatic loop to be stationary while the edge dislocation moves on its glide plane. For a fixed loop orientation, Kroupa [10] and Makin [11] have analyzed the interaction with a straight dislocation in considerable detail.

For the present case, the traction vector $\mathbf{t}$ has only two components, namely

$$
t_{x}=b \square_{x x}=H \frac{\left(1+3 \square^{2}\right)}{\left(1+\square^{2}\right)^{2}} \text { and } t_{y}=b \square_{x y}=H \frac{\square\left(1 \square \square^{2}\right)}{\left(1+\square^{2}\right)^{2}} \text {, with } H=\frac{\square b^{2}}{2 \square(1 \square \square) h} \text { and } \square=\frac{x}{h}
$$

We see that $t_{y}$ changes its sign, and it, as well as $\square$ and $\square$, become negative for $\square>1$. Equation (15) takes then the form

$$
\operatorname{sign}(1 \square \square) \sqrt{k(1 \square k)} \square(k)=H \frac{\square\left(1 \square \square^{2}\right)}{\left(1+\square^{2}\right)^{2}}
$$

Its numerical solution, for the particular set of parameters stated in the caption, is shown in Figure 2. Several points are noteworthy. First, we see very abrupt changes or flips in the loop orientation at the midplane, $x=0$, and at the diagonal planes, $x= \pm y$. Second, the dislocation loop remains rotated away from its equilibrium orientation $\square=0$ even at far distances from the edge dislocation where its stress field is weak.

The interaction energy between loop and edge dislocation consists of two parts, namely the change in the self-energy of the loop as it is rotated by the stress field of the edge dislocation, and the interaction proper with the stress field. Using the rotation angle $\square(\square)$ from the solution of equation (17), the glide force $F$ as defined in equation (6) is obtained. Since the gradient of stress fields for straight dislocation diminishes as $r^{-2}$ where $r$ is the radial distance from the dislocation, we display the quantity 
[ 2(1- $\left.\square / \square b^{2}\right]\left(r / R_{0}\right)^{2} F$ in Figure 5. The solid curve shows this normalized glide force when the loop is allowed to adjust its orientation, while the dashed curve shows the classical result $[10,11]$ for the same loop with orientation held fixed at $\square=0$. The rotation caused by the stress is seen to significantly enhance the glide force at large distances. Although the force is weak in absolute terms at these large distances, it is there where this force begins to impose a drift on the otherwise random motion of the loop, leading to its eventual capture in close vicinity of the dislocation.

In summary, we have derived the complete and consistent expressions for glide force and torque exerted by an arbitrary stress field on a glissile dislocation loop, and for the selftorque of the loop. A solution has been obtained for the loop orientation as a function of its location, and this solution is employed to derive the actual interaction energy or glide force for the combined motion and rotation of a loop in a general stress field. We have demonstrated that stress-induced rotation can significantly enhance the glide force. We gratefully acknowledge the support of this work by a NERI grant from the U.S. Department of Energy with the Lawrence Livermore National Laboratory under contract W-7405-Eng-48.

\section{References}

[1] Yu.N. Osetsky, D.J. Bacon, A. Serra, B.N. Singh, S.I. Golubov, J. Nucl. Materials 276 (2000) 65

[2] J. Marian, B.D. Wirth, A. Caro, B. Sadigh, G.R. Odette, J.M. Perlado, T. Diaz de la Rubia, Phys. Rev. B65 (2002) 144102

[3] F. Kroupa, Theory of Crystal Defects; Proceedings, ed. B. Gruber, Acad. Press, New York, 1966, p. 275

[4] N.M. Ghoniem, B.N. Singh, L.Z. Sun, T. Diaz de la Rubia, J. Nucl. Materials 276 (2000) 166

[5] P.P. Gillis and J. Kratochvil, Phil. Mag. 20 (1970) 425

[6] R. Bullough and A.J.E. Foreman, Phil. Mag. 9 (1964) 315

[7] J.C.M. Li and G.C.T. Liu, Phil. Mag. 14 (1966) 413

[8] D.J. Bacon, Phil. Mag. 14 (1966) 715

[9] R. de Wit and A.W. Ruff, Jr., Phil. Mag. 15 (1967) 1065

[10] F. Kroupa, Phil. Mag. 7 (1962) 783

[11] M.J. Makin, Phil. Mag. 10 (1964) 695 


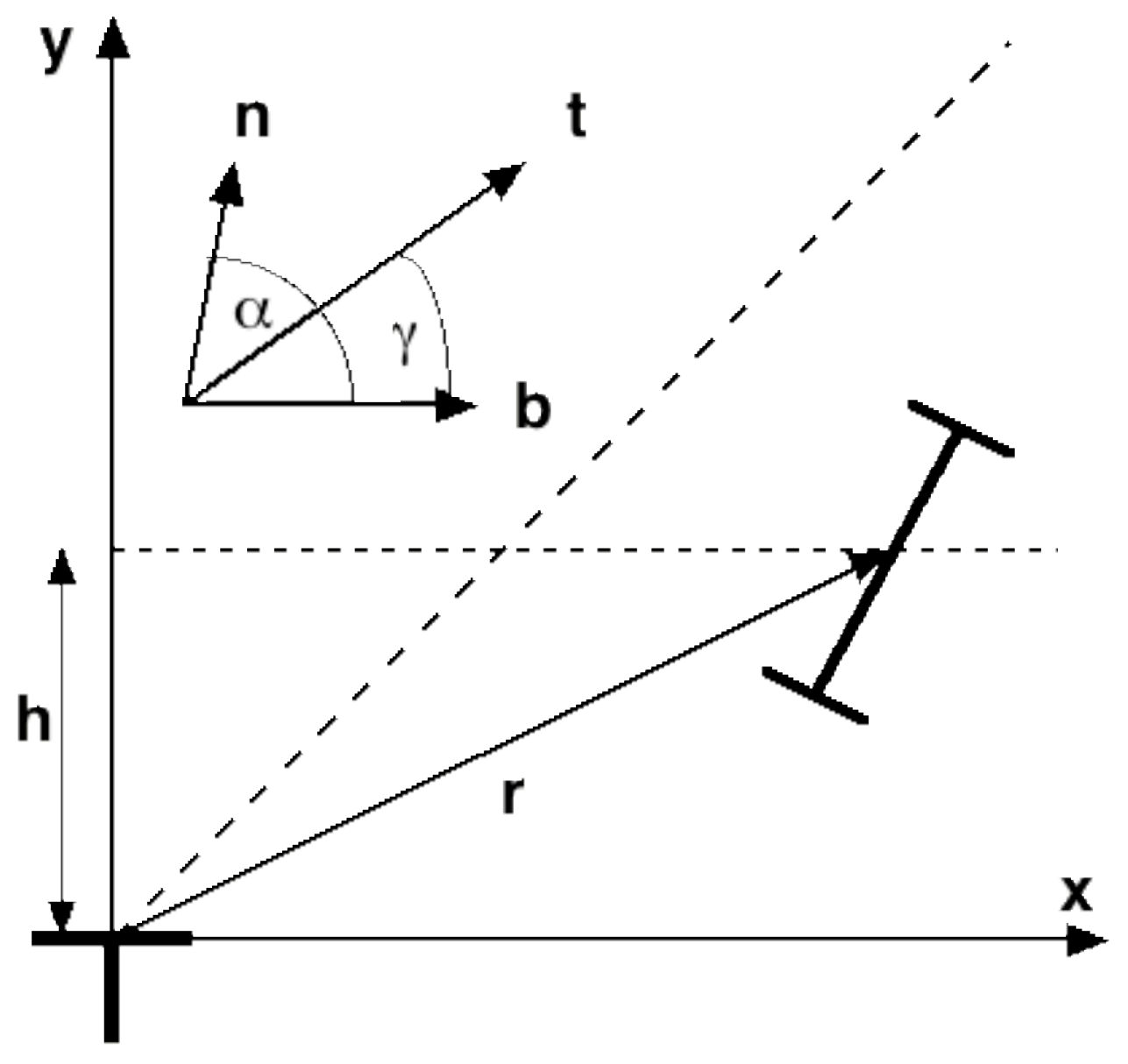

Figure 1. The insert shows the three vectors required to characterize the orientation of a prismatic, interstitial-type loop. The Burger vector $\mathbf{b}$ which is given and determines the glide direction. The traction vector field $\mathbf{t}(\mathbf{r})$ which is defined by the source of stress, here an edge dislocation, and the normal vector $\mathbf{n}$. As the loop glides along its glide cylinder, parallel to the dotted line, the orientations of $\mathbf{t}$ and $\mathbf{n}$ change. 


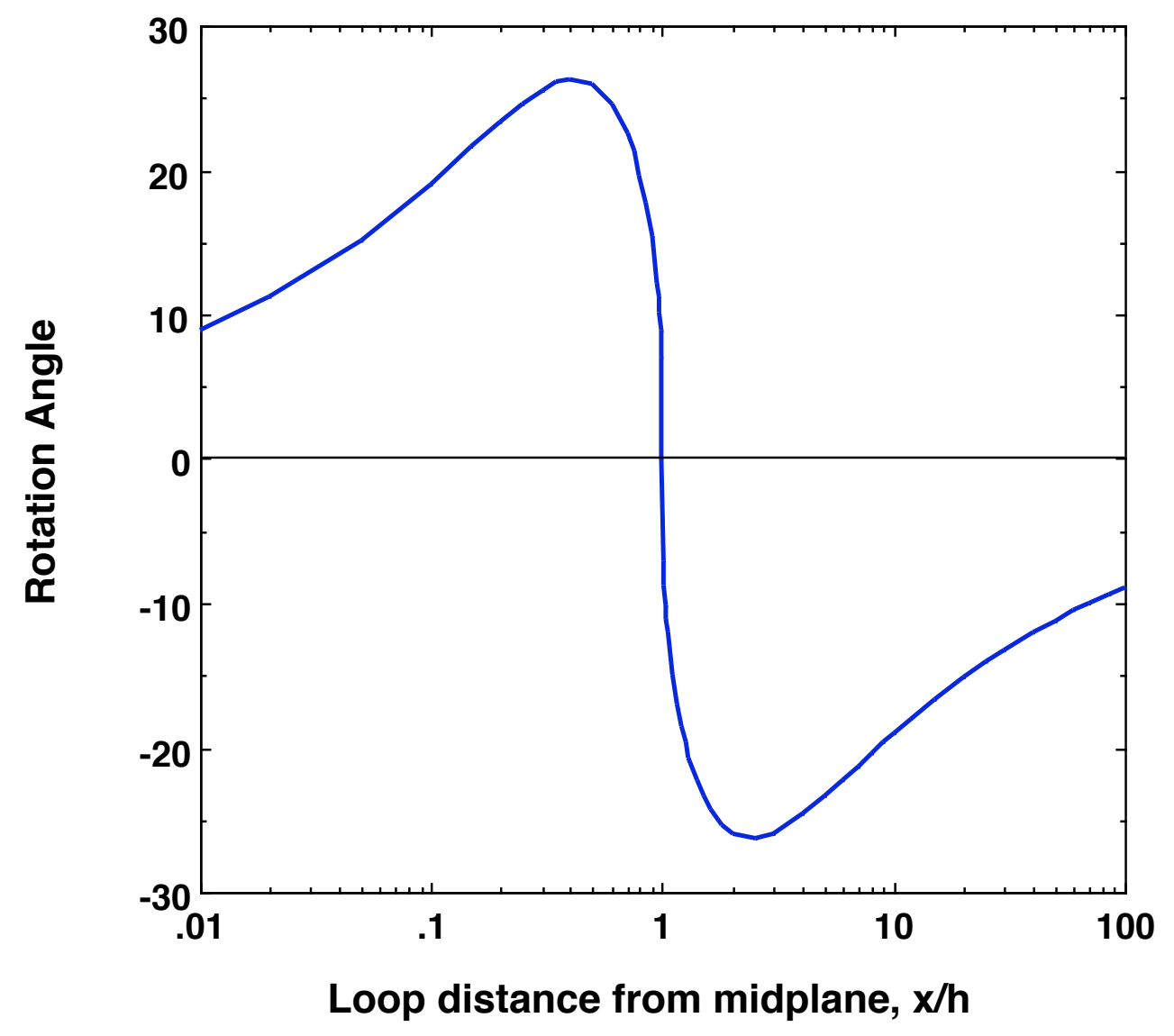

Figure 2. The rotation angle $\square$ as a function of the loop position along its glide path. The results shown here and in the next figure are for a loop with a radius of $R_{0}=5 r_{0}$, and for a distance $h=3 R_{0}$ from the glide plane of the edge dislocation. 


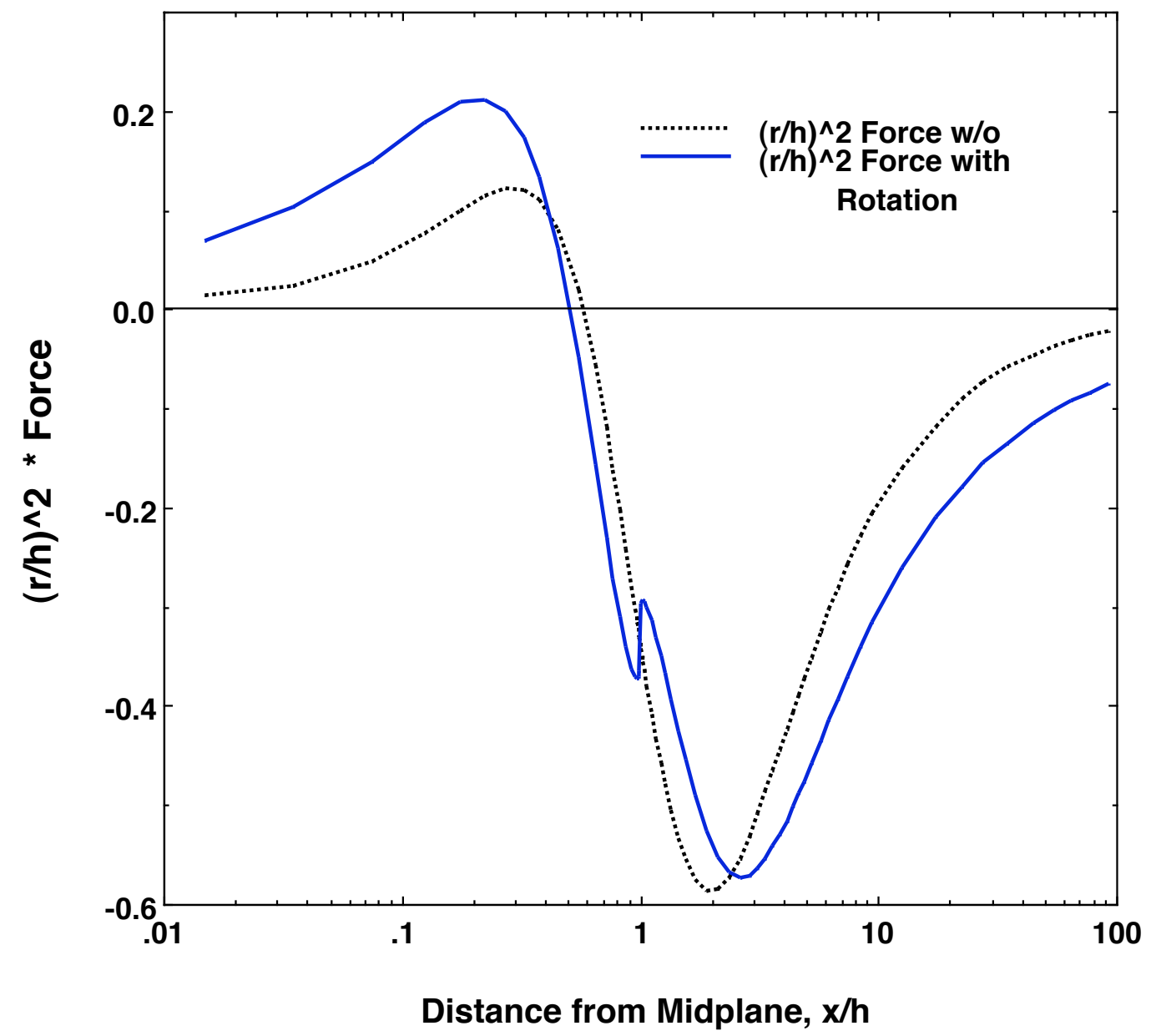

Figure 3. The normalized glide force (see text for details) exerted by an edge dislocation on a prismatic interstitial-type loop moving parallel to the glide plane of the dislocation. Dotted curve shows this force when the loop orientation remains fixed, the solid curve when the loop rotates such that the applied torque is balanced by the loop's self-torque. 
